\title{
Roles of VRK1 as a new player in the control of biological processes required for cell division
}

\author{
Alberto Valbuena, Marta Sanz-García, Inmaculada López-Sánchez, Francisco M. \\ Vega, and Pedro A. Lazo*,
}

Experimental Therapeutics and Translational Oncology Program, Instituto de Biología Molecular y Celular del Cáncer, Consejo Superior de Investigaciones Científicas (CSIC) - Universidad de Salamanca, Campus Miguel de Unamuno, E-37007 Salamanca, Spain

Running title: VRK1 coordination of cell division

Key words: cell cycle, kinase, p53, Golgi, nuclear envelope, histone, BANF1, chromatin

*Correspondence to: P.A. Lazo, IBMCC-Centro de Investigación del Cáncer, CSICUniversidad de Salamanca, Campus Miguel de Unamuno, E-37007 Salamanca, Spain. Phone: (34) 923294 804, Fax (34) 923294 795. E-mail: pedro.lazo@csic.es 


\begin{abstract}
Cell division, in addition to an accurate transmission of genetic information to daughter cells, also requires the temporal and spatial coordination of several biological processes without which cell division would not be feasible. These processes include the temporal coordination of DNA replication and chromosome segregation, regulation of nuclear envelope disassembly and assembly, chromatin condensation and Golgi fragmentation for its redistribution into daughter cells, among others. However, little is known regarding regulatory proteins and signalling pathways that might participate in the coordination of all these different biological functions. Such regulatory players should directly have a role in the processes leading to cell division. VRK1 (Vaccinia-related kinase 1) is an early response gene required for cyclin D1 expression, regulates p53 by a specific Thr18 phosphorylation, controls chromatin condensation by histone phosphorylation, nuclear envelope assembly by phosphorylation of BANF1, and participates in signalling required for Golgi fragmentation late in the G2 phase. We propose that VRK1, a Ser-Thr kinase, might be a candidate to play an important coordinator role in these cell division processes as part of a novel signalling pathway.
\end{abstract}

\title{
1. VRK1 and mitotic processes.
}

Cell division is a complex process requiring the synchronization of multiple functions in addition to a correct DNA replication and chromosome segregation in mitosis [1]. Several cellular processes have to be coordinated to generate two daughter cells. These include nuclear membrane disassembly and reassembly, chromatin condensation, mitotic spindle assembly and disassembly, centrosome duplication, cyclin gene expression, Golgi fragmentation, and setting up DNA damage response pathways in a readiness state to intervene if necessary by acting on mitotic checkpoints. All of them need to be coordinated, otherwise cell division will not be feasible, but so far no candidate regulatory protein or signalling pathway has been clearly identified for this role. Such coordinator protein must be able to receive and integrate signals with different origins such as growth factors or cell damage. This protein must also have downstream targets that have to be implicated and be essential in several of the processes to be regulated, in which this protein will also function as a master coordinator. To perform such coordinator role, a kinase would be the most suitable 
candidate since kinases are able to receive input signals from different origins and, their action is reversible and they can have multiple substrates. These substrates would have to include key components of the different processes targeted and involved in cell division, many of which are known to be regulated by different kinases either being directly phosphorylated or requiring the participation of a kinase for their function.

In this context we hypothesize that the kinase VRK1 is a candidate to perform such a coordinating role, based on current data available, since it is required to exit G0 and enter in the G1 phase of cell cycle [2], has several targets affecting progression of G1 phase [2, 3]and participates in nuclear envelop dynamics [4], chromatin condensation [5] and Golgi fragmentation [6] (Fig. 1). The functional position of such coordinator protein affecting several processes suggests that this gene is not likely to be mutated in cancer, since its consequences are likely to have catastrophic and cells will not be viable, in case of loss of function. In a large study searching for kinase mutations in human cancer, VRK1 is not mutated, consistent with such a role [7].

\section{The human VRK1 Ser-Thr kinase}

In the human kinome there is an isolated and early divergent branch from the CK1 family, the VRK (Vaccinia-related kinase) family of Ser-Thr kinases, which is composed of three members in mammals [8], and of which only two, VRK1 and VRK2 are active as kinases $[9,10]$. These VRK proteins have aminoacid substitutions in key residues that are conserved in almost all other kinases in the human kinome [8]. VRK1 consists of 396 aminoacids with an N-terminal active kinase domain. Its C-terminal region has a nuclear localization signal, and a basic-acid-basic motif (BAB motif) of unknown function. VRK1 does not have any additional distinctively known feature that might be present in other proteins [11]. The VRK1 protein is mostly located in the nucleus, but it is also present in Golgi and some cytosolic vesicles [6, 12], and the regulation of its subcellular distribution is not yet known.

The VRK family has a unique ortholog gene in C. elegans, Vrk-1[13] and $D$. melanogaster, NHK-1 (nucleosomal histone kinase-1) [14, 15]. The homology is restricted to their kinase domain, and all of them have C-terminal regions which are unrelated among them, and with few known characteristics. These structural differences suggest that they might have common kinase substrates, but probably different regulation or protein interactions. No ortholog has been identified in yeasts. This situation resembles that of the p53 protein, which is not present in yeasts and that also 
appeared late in evolution with a single member in lower eukaryotes (invertebrates) and three members in mammals $[16,17]$. These data suggests that VRK and p53 protein families could have a parallel evolution.

\section{VRK1 expression is associated with proliferation phenotype in tumours}

Any protein that might control cell division processes must be associated with the proliferation phenotype. Thus, if VRK1 is a candidate for such a role it must be expressed, probably at high levels, in proliferating cells. Several studies on VRK1 gene expression and protein levels in different systems indicate a potential role for VRK1 in cell proliferation. Initially high VRK1 RNA levels were detected in human tumour cells lines and normal tissues with high proliferation rates [18], and also in murine thymus and testis [19]. Also, during murine embryonic hematopoietic development, VRK1 expression reached its peak at days E11.5 to E13.5, a time at which there is a massive embryonic cellular expansion [19]. In normal human epithelia, high VRK1 protein level was mostly detected in the transit amplifying compartment, colocalizing with p63 [2].

VRK1 protein expression has also been determined in different human cancers. In head and neck squamous cell carcinomas (HNSCC), high VRK1 protein level positively correlated with several proliferation markers including Ki67, p63, CDK2,

CDK6, cdc2, cyclin A, cyclin B1, topoisomerase II and survivin [20]. Lung cancers also have high VRK1 levels, which are more pronounced in those tumours with p53 mutations [21]. In breast cancer, VRK1 is included within the sixteen-kinase gene expression signature reflecting a high mitotic activity, and which identifies luminal breast cancers with a poorer prognosis [22]. In human ER+ breast cancer, VRK1 overexpression is also a predictor of poor response [23], and its downregulation is an indicator of a good outcome [24]. Tumour cell lines also express high levels of VRK1 protein [21], and knock down of VRK1 results in a stop of cell proliferation in tumour [25] and fibroblasts cell lines [2].

\section{VRK1 gene is regulated in cell cycle and necessary for its progression}

A protein coordinating cell division must be implicated in cell cycle entry. VRK1 is necessary for cell cycle entry [2]. In this context, VRK1 gene expression is turned off by serum withdrawal, resulting in a block in cell cycle progression, and serum readdition induces an immediate reexpression of the VRK1 gene [2]. VRK1 gene expression parallels that of MYC and FOS, behaving like an early gene [2] and 
coincides with detectable expression of cyclin D1 gene (CCND1), and precedes that of cyclin E (CCNE1) [2]. VRK1 knockdown results in loss of Rb phosphorylation [2], consistent with its early role in cell cycle progression. This early role is also supported by the identification of VRK1 protein within the transcriptional complex required for CCND1 gene expression by phosphorylation in Ser133 of the CREB transcription factor in G1/S [3]. Changes in VRK1 protein level are also positively correlated with phospho-Rb, PCNA, and cyclinB1 and negatively with p27 [2]. Following serum withdrawal there is a drop of VRK1 and PCNA and an increase in p27 and dephosphorylated Rb levels [2]. Also Rb knockdown induces an increase of VRK1 gene expression [26], thus confirming the regulation of VRK1 in cell cycle progression. This early activation of VRK1 expression is detected as an increase in VRK1 protein levels as cell cycle progresses, which is higher at the $\mathrm{S}$ phase [5]. Furthermore, in cancers driven by mutation of KRAS, VRK1 and TBK1 are required for their progression [27]. Thus VRK1 can control cell cycle progression at an early stage, and might even have different roles depending on protein levels and activation in different cell cycle phases.

Certain stem cells have been proposed to follow a particular type of cell division, asymmetric division, that [28] generates two different daughter cells. One will remain with stem cell characteristics and the other will become a proliferating precursor that eventually will differentiate. VRK1 is upregulated in the embryonic stem and forms part of the asymmetric signature cell module that guides creation of epithelial cancer stem cells [29]. Also in salamander, an animal model for regeneration, expression of the VRK1 ortholog gene is upregulated during natural spinal cord regeneration [30].

\section{VRK1 and DNA synthesis}

The initiation of DNA synthesis is a key event in cell division, but the role of VRK1 on DNA in this process has not yet been directly addressed. However, there is some indirect evidence suggesting it might play an important role. In cell cycle, VRK1 levels are higher in the S phase [5], and overexpression of human VRK1 induces an increase in the incorporation of bromodeoxyuridine into DNA, suggesting cells enter the S phase [3]. But these changes might be an indirect consequence of VRK1 promotion of cell cycle progression, and not a consequence of a direct effect on DNA replication by itself. Also Vaccinia virus B1R protein, which has a $39 \%$ identity and gave the name to the VRK protein family, is necessary for viral DNA replication, and 
this viral function can be partially rescued by VRK1, and VRK2, in vaccinia viruses that are deficient in B1R function [31].

\section{VRK1 and p53, the key player in protection against genetic damage}

Cells require precise safeguards mechanisms to protect them from DNA damage, which are required to guarantee fidelity in transmission of genetic information. The tumor suppressor p53 is a key player in the cellular response to stress and genotoxic damage, participating in cell cycle checkpoints [32-35]. The functions of p53 in response to DNA damage are regulated by phosphorylation of several residues in its $\mathrm{N}$ terminal transactivation domain and many kinases have been implicated in this regulation [36, 37]. VRK1 phosphorylates p53 uniquely in Thr18 [9, 25]. This Thr18 residue forms a hydrogen bond with Asp21 that is necessary to maintain an hydrophobic side in the p53 alpha helix needed for interaction with its negative regulator mdm2 [38]. Disruption, by phosphorylation, of the p53 alpha helix destabilizes its structure and results in loss of binding to a hydrophobic pocket in mdm2 [38]. Thr18 is the key residue controlling the p53 switch from binding to mdm2 [39] to interaction with transcriptional cofactors [40, 41]. Phosphorylation in Thr18 switches p53 to a binding mode for transcriptional cofactors, and additional phosphorylations will modulate an increase in either specificity or affinity for one cofactor or another [40,41], thus determining the final specific biological effect, among the many responses triggered by an activated p53 [42, 43].

Normal cells must have a basal level of p53 that is ready to respond immediately to stimulation induced by cellular stress or DNA damage. This p53 stabilization is mediated by phosphorylation in Thr18 mediated by VRK1, this active phosphorylated p53 initiates an apoptotic or cell cycle stoppage response depending on additional signals received from other pathways [25], a phosphorylation mediated by other kinases in Ser15 and Ser20, that have a secondary role in the switch, these additional phosphorylations are critical for determining the selection and interaction of p53 with transcriptional cofactors [40]. But a stable accumulation of phosphorylated p53 will result in a permanent block, which will make a cell non viable or in cell death. Thus p53 levels must be tightly regulated and should fluctuate depending on conditions. The p53 stabilization has to be reversible and that is the role of mdm2, which downregulates p53 by its ubiquitylation and subsequent degradation in the proteasome [44]. The requirement of mdm2 expression is a late event compared with the immediate 
consequence of p53 phosphorylation, thus p53 must have oscillations in its level to permit repair without deleterious effects, which have been identified based on p53mdm2 pair [45, 46]. However, there are additional components in the regulatory process. For example, p53 needs to be dephosphorylated to be able to interact with mdm2, but although some p53 phosphatases such as Wip1 [28] and PP1 [47] have been identified, they have not been fully studied and their integration in the temporal regulation of p53 remains to be completely understood.

VRK1 is a stabilizer of p53 by its Thr18 specific phosphorylation, but this phosphate has to be removed to permit p53 binding and ubiquitylation by mdm2, and one way is to remove its stabilizer. The p53 induced downregulation of VRK1 is indirect and does not affect VRK1 gene promoter or VRK1 ubiquitylation by mdm2 [48]. Recent experiments have demonstrated that VRK1 is downregulated in a p53dependent manner in the autophagic pathway and protein degradation in the lysosome [48]. The accumulation of phosphorylated p53 induces the gene expression of DRAM [49]. DRAM is a small protein with four transmembrane domains and located in endosomes, lysosomes and other vesicles. DRAM is the likely mediator that targets VRK1 for lysosomal degradation. VRK1 degradation in lysosomes is eliminated by either knockdown of DRAM or of Beclin1, suggesting VRK1 is degraded by the autophagic pathway [50]. The removal of VRK1 permits p53 dephosphorylation, and thus p53 becomes accessible to mdm2 and subsequent degradation in the proteasome. Thus VRK1 and p53 can both be downregulated [48] relieving the block and preventing deleterious consequences of stable high levels of p53 (Fig.3). The VRK1-p53 relation appeared early in evolution; in C. elegans, also vrk-1 appears to regulate CEP-1(p53) controlling germ-cell proliferation [51].

\section{VRK1 implication in the control of nuclear membrane dynamics}

In mitosis, disassembly of the nuclear envelope is a process necessary to permit chromosome segregation into daughter cells and cell cycle progression. An important step in cell division is represented by the disassembly and reassembly of nuclear envelope, which is associated to chromatin condensation early in mitosis and after the end of chromosome segregation late in mitosis. Thus the nuclear membrane has a temporal and spatial regulation [52]. In this process BANF1 (barrier to autointegration factor 1) plays an important role. BANF1 is a small $10 \mathrm{kDa}$ protein that forms dimers and interacts with DNA in a non-sequence specific manner [53]. BANF1 also binds to 
proteins containing LEM domains, present in the inner nuclear membrane [54]; by this interaction BANF1 anchors DNA in interphase to the nuclear membrane and participates in chromatin remodelling complexes [52]. The effects of BANF1 are regulated by phosphorylation. VRK1 phosphorylates the N-terminal domain of BANF1 in residues Ser4 or Thr2/Thr3, and VRK1 overexpression reduces BANF1 interaction with nuclear chromatin resulting in its dispersal and disassembly [4]. Phosphorylated BANF1 also has a reduced binding to DNA and LEM-containing proteins [4], delocalizing emerin and interfering with emerin binding to lamin A, both in mitosis and interphase [55]. The phosphorylation of BANF1 by VRK1 might be dependent on functional changes in the activity of VRK1, a kinase that is regulated during cell cycle in expression and activity, and required for G0 exit and G1 progression [2]. Also D. melanogaster NHK1 [56] and C. elegans Vrk-1 ortholog proteins [13] phosphorylated BANF1 and affect the structure of the nuclear membrane. Since the conservation among VRK1 proteins from three species is restricted to their kinase domain, BANF1 phosphorylation by VRK1 was acquired as an early functional role in the evolution of this kinase.

\section{Regulation of VRK1 activity by interaction with the Ran small GTPase}

The enzymatic activity of VRK1 can be regulated by different mechanisms that can affect either its kinase activity or its substrate specificity. These regulatory mechanisms include either protein interactions or covalent modifications. The first allosteric regulator of VRK proteins identified is the small GTPase Ran, which is the only nuclear member of the large small GTPase family [57]. Ran stably interacts with the three VRK proteins, but only the Ran interaction with VRK1 has been characterized [58]. Small GTPases have two alternative forms depending on the bound nucleotide; inactive or bound to GDP, and active or bound to GTP. Ran-GDP is mostly located in the cytosol and participates in the nuclear transport mechanism, kinetochore function [59], spindle assembly [60] and mitosis [61]; Ran-GTP, is located in the nucleus, and the nucleotide exchange from Ran-GDP to Ran-GTP is mediated by the nuclear GEF (Guanine exchange factor) RCC1 [62]. During mitosis Ran-GTP can form a concentration gradient, with the highest concentration near condensed chromosomes $[61,63]$. If generation of Ran-GTP is blocked there is no formation of a mitotic spindle and chromosomes are misaligned [62]. Ran-GDP, but not Ran-GTP inhibits the kinase activity of VRK1 [58]. Although the full consequences of this VRK1 regulation are not 
known, it has two clear effects. First, the active VRK1-Ran-GTP complex near chromosomes might facilitate phosphorylation of histones and chromatin condensation; and second the inactive VRK1-Ran-GDP complex might facilitate VRK1 nuclear transport and probably has additional roles not yet identified.

\section{Histone phosphorylation by VRK1 and chromatin condensation}

One of the initial events in mitosis after DNA replication is chromatin condensation which requires covalent modification of histones, particularly in the tail of H3 [64, 65]. Some histones are also substrates of VRK proteins. Drosophila melanogaster NHK1 (nucleosomal histone kinase) phosphorylates mitotic histones H2A which is required for acetylation of $\mathrm{H} 3$ [14, 66, 67], a phosphorylation also required for mitotic progression [15]. VRK1 contributes to chromatin condensation by phosphorylation of histone H3 [5, 58]. Human VRK1 directly phosphorylates histone H3 in Ser10 resulting in chromatin condensation, and cooperates with Aurora B, a process also required for progression of mitosis [5]. Ran-GTP binds to VRK1 without altering its activity, but Ran-GDP binding to VRK1 inhibits its kinase activity [58]. Since there is a nuclear gradient of Ran-GTP, this permits the formation of a nuclear gradient of VRK1 activity in the nucleus depending on its partner interactions. VRK1 will be active near the chromosomes, where it can participate in chromatin condensation by phosphorylation of histone H3 [5], a phosphorylation that is lost by knocked-down of VRK1 [58]. Histone H3 can also be phosphorylated in Ser10 by Aurora B [68], and it is likely that VRK1 and Aurora B might be part of a common pathway not yet defined.

In interphase, VRK1 colocalizes with heterochromatin and interacts with gamma heterochromatin protein 1 (HP1 $\gamma$ ) [5]; while in a dividing cell, after chromatin condensation, VRK1 is not bound to chromosomes and appears dispersed with a granular aspect, where it might be participating in different signalling pathways not yet identified (Fig.2). Thus, VRK1 might have different roles in different phases of the cell cycle.

\section{VRK1 in Golgi fragmentation}

In cell division cytoplasmic organelles also have to be distributed in daughter cells. But for unique organelles as the Golgi fragmentation in smaller vesicles is a necessary step during mitosis so that they can be redistributed into daughter cells during the G2/M phase [69]. The regulation of Golgi fragmentation is not well known, 
but mitogenic signals mediated by MAP kinases, particularly MEK1, induce Golgi fragmentation [70-72], representing an upstream component of the fragmentation pathway responding to a mitogenic signal. This mitogenic signal has a cytosolic branching point, in which downstream of MEK1 this fragmentation signal is mediated by Plk3 (Polo-like kinase 3) [73], a member of the Plk (polo-like kinase) protein family [74].

There is a subpopulation of VRK1 protein in the cytosol that colocalizes with several markers of the Golgi apparatus [12], such as giantin or GM130 [6]. This colocalization suggested that VRK1 might participate in processes regulating Golgi functions, and fragmentation in mitosis is a likely candidate. VRK1 has a consensus sequence for phosphorylation by Plk proteins and Plk3 phosphorylates VRK1 in Ser342 within this sequence [6]. Mutation of this residue in VRK1, knocking-down VRK1, or the use of a catalytically inactive VRK1 ${ }^{\mathrm{K} 179 \mathrm{E}}$, all block Golgi fragmentation induced by both MEK1 and Plk3 [6]. Thus, one of the roles of the cytosolic VRK1 subpopulation late in G2 is to contribute to Golgi fragmentation in G2/M during mitosis [75, 76], and VRK1 is thus a new downstream step of this pathway in mitosis. In this process the situation of VRK1 downstream of MEK1-PIk3 permits that other signals mediated by MEK1 can only be transmitted to VRK1 at a time point when there is a significant level of VRK1 in the cytoplasm; thus the level of VRK1 expression and its subcelullar localization can contribute to the timing of specific events in cell division.

\section{Phenotypes associated to loss of VRK expression in vivo: defects in proliferation}

Initial studies reporting phenotypes due to mutation or loss of VRK proteins were made in model organisms and the consequences range from embryo lethality to sterility. In D. melanogaster, a hypomorphic mutation in $n h k-1$ results in male and female sterility due to defective formation of the mitotic spindle in meiosis [77]. Other mutations in $n h k-1$ cause aberrant mitotic spindle in mitosis, and more severe $n h k-1$ mutations are embryonic lethal [15]. In C. elegans, loss of vrk-1 by RNAi is also embryonic lethal, and there are failures in cell division affecting chromatin organization and mitotic spindle [78], as well as defects in nuclear pore formation [79]. In adults its silencing results in a slow growth phenotype [78]. Also Vrk-1 mutations in germinal cells result in inability to form gametes and sterility [80]. In C. elegans proliferation 
defects in Vrk-1 can be rescue by CEP-1/p53, suggesting and early relationship between VRK1 and p53 [51].

In murine models, no viable knockout mice has been generated, but there are some data obtained in a hypomorphic gene trap knock-out model of murine VRK1, with a residual $15 \%$ expression. There were described defects in gamete formation, affecting spermatogonia and oocyte formation, which lead to male and female sterility [81-83].

In humans, a homozygous mutation affecting the nuclear localization signal of VRK1 causes a rare muscular atrophy syndrome with pontocerebellar hypoplasia, ataxia and muscular atrophy [84]. This rare mutation suggests that VRK1 might also have a role in neural development.

These results suggest the importance of VRK1 in regulation of cell division and development, and it is likely than a knock-out model might be embryonic lethal. Conditional knock-out models addressing specific questions have not yet been reported.

\section{Is there a cell division coordinator role for VRK1?}

All the evidence currently available points to a potential role for VRK1 in coordinating different processes required for cell division, although still many issues remain to be clarified. A protein that integrates signals coordinating the different biological processes needed for a successful cell division has to participate in all of these processes (Figure 3). VRK1 is regulated in cell cycle, regulates p53, the main regulator of stress responses and DNA damage, participates in nuclear envelope assembly and disintegration, and Golgi fragmentation. If this coordinating role is real, VRK1 should also be activated by different types of stimuli that affect cell division, independently of their origin, thus all types of DNA damage should activate VRK1, as well as several growth factors that induce entry in cell cycle. However, additional components of the VRK1 system must be found in order to identify the response to the initiating signal, as well as downstream targets to connect with specific processes. Presumably the integration of VRK1 in macromolecular complexes would allow it to perform such a variety of functions and determine the specificity.

Current therapies in oncology are targeted at individual steps of processes require for the tumor cell, demanding the use of combination protocols with drugs targeting different proteins. An alternative would be to target a protein that can affect simultaneously several cellular processes, making it likely that the cell enter in a 
catastrophic situation and become non-viable. Theoretically a coordinator protein might be a good candidate for therapeutic development applied to processes where proliferation plays an important role, particularly in the case of kinases for which it might be feasible to design specific inhibitors. Inhibition of VRK1 will target several of the hallmarks of the cancer phenotype [85]. For this reason VRK1 might be a suitable target for therapeutic development as divergences between VRK1 catalytic domain and the one of other kinases can facilitate the development of kinase-specific inhibitors [86, 87], which might be helpful in cancer therapy, but this is something that needs further exploration. Also an increased understanding about VRK kinases biology and their mode of action would allow targeted therapies that interfere with its function in cancer cells without the possible physiological consequences of manipulating such a central signalling mediator.

\section{Acknowledgements}

The laboratory is supported by grants from Ministerio de Ciencia e Innovación (SAF201014935 and CSD2007-0017), Junta de Castilla y León (Consejería de Educación, GR15), and Kutxa-Fundación Inbiomed.

\section{REFERENCES}

[1] Olashaw N, Pledger WJ, Sci STKE. 2002;2002:RE7.

[2] Valbuena A, Lopez-Sanchez I, Lazo PA, PLoS ONE. 2008;3:e1642.

[3] Kang TH, Park DY, Kim W, Kim KT, J. Cell. Sci. 2008;121:3035-3041.

[4] Nichols RJ, Wiebe MS, Traktman P, Mol. Biol. Cell. 2006;17:2451-2464.

[5] Kang TH, Park DY, Choi YH, Kim KJ, Yoon HS, Kim KT, Mol. Cell. Biol. 2007;27:8533-8546.

[6] Lopez-Sanchez I, Sanz-Garcia M, Lazo PA, Mol. Cell. Biol. 2009;29:1189-1201. [7] Greenman C, Stephens P, Smith R, Dalgliesh GL, Hunter C, Bignell G, Davies H, Teague J, Butler A, Stevens C, Edkins S, O'Meara S, Vastrik I, Schmidt EE, Avis T, Barthorpe S, Bhamra G, Buck G, Choudhury B, Clements J, Cole J, Dicks E, Forbes S, Gray K, Halliday K, Harrison R, Hills K, Hinton J, Jenkinson A, Jones D, Menzies A, Mironenko T, Perry J, Raine K, Richardson D, Shepherd R, Small A, Tofts C, Varian J, Webb T, West S, Widaa S, Yates A, Cahill DP, Louis DN, Goldstraw P, Nicholson AG, Brasseur F, Looijenga L, Weber BL, Chiew YE, DeFazio A, Greaves MF, Green AR, Campbell P, Birney E, Easton DF, Chenevix-Trench G, Tan MH, Khoo SK, Teh BT, Yuen ST, Leung SY, Wooster R, Futreal PA, Stratton MR, Nature. 2007;446:153-158. [8] Manning G, Whyte DB, Martinez R, Hunter T, Sudarsanam S, Science (New York, N.Y. 2002;298:1912-1934.

[9] Lopez-Borges S, Lazo PA, Oncogene. 2000;19:3656-3664. 
[10] Nichols RJ, Traktman P, J. Biol. Chem. 2004;279:7934-7946.

[11] Klerkx EP, Lazo PA, Askjaer P, Histol. Histopathol. 2009;24:749-759.

[12] Valbuena A, Lopez-Sanchez I, Vega FM, Sevilla A, Sanz-Garcia M, Blanco S, Lazo PA, Arch. Biochem. Biophys. 2007;465:219-226.

[13] Gorjanacz M, Klerkx EP, Galy V, Santarella R, Lopez-Iglesias C, Askjaer P, Mattaj IW, Embo J. 2008;26:132-143.

[14] Aihara H, Nakagawa T, Yasui K, Ohta T, Hirose S, Dhomae N, Takio K, Kaneko

M, Takeshima Y, Muramatsu M, Ito T, Genes Dev. 2004;18:877-888.

[15] Cullen CF, Brittle AL, Ito T, Ohkura H, J. Cell. Biol. 2005;171:593-602.

[16] Belyi VA, Ak P, Markert E, Wang H, Hu W, Puzio-Kuter A, Levine AJ, Cold

Spring Harb. Perspect. Biol. 2010;2:a001198.

[17] Dötsch V, Bernassola F, Coutandin D, Candi E, Melino G, Cold Spring Harbor Perspectives in Biology. 2010;2, a004887.

[18] Nezu J, Oku A, Jones MH, Shimane M, Genomics. 1997;45:327-331.

[19] Vega FM, Gonzalo P, Gaspar ML, Lazo PA, FEBS Lett. 2003;544:176-180.

[20] Santos CR, Rodriguez-Pinilla M, Vega FM, Rodriguez-Peralto JL, Blanco S,

Sevilla A, Valbuena A, Hernandez T, van Wijnen AJ, Li F, de Alava E, Sanchez-

Cespedes M, Lazo PA, Mol. Cancer Res. 2006;4:177-185.

[21] Valbuena A, Suarez-Gauthier A, Lopez-Rios F, Lopez-Encuentra A, Blanco S,

Fernandez PL, Sanchez-Cespedes M, Lazo PA, Lung Cancer. 2007;58:303-309.

[22] Finetti P, Cervera N, Charafe-Jauffret E, Chabannon C, Charpin C, Chaffanet M, Jacquemier J, Viens P, Birnbaum D, Bertucci F, Cancer research. 2008;68:767-776.

[23] Martin KJ, Patrick DR, Bissell MJ, Fournier MV, PLoS ONE. 2008;3:e2994.

[24] Fournier MV, Martin KJ, Kenny PA, Xhaja K, Bosch I, Yaswen P, Bissell MJ, Cancer research. 2006;66:7095-7102.

[25] Vega FM, Sevilla A, Lazo PA, Mol. Cell. Biol. 2004;24:10366-10380.

[26] Semizarov D, Kroeger P, Fesik S, Nucleic Acids Res. 2004;32:3836-3845.

[27] Barbie DA, Tamayo P, Boehm JS, Kim SY, Moody SE, Dunn IF, Schinzel AC, Sandy P, Meylan E, Scholl C, Frohling S, Chan EM, Sos ML, Michel K, Mermel C, Silver SJ, Weir BA, Reiling JH, Sheng Q, Gupta PB, Wadlow RC, Le H, Hoersch S, Wittner BS, Ramaswamy S, Livingston DM, Sabatini DM, Meyerson M, Thomas RK, Lander ES, Mesirov JP, Root DE, Gilliland DG, Jacks T, Hahn WC, Nature.

2009;462:108-112.

[28] Fiscella M, Zhang H, Fan S, Sakaguchi K, Shen S, Mercer WE, Vande Woude GF, O'Connor PM, Appella E, Proc. Natl. Acad. Sci. U S A. 1997;94:6048-6053.

[29] Wong DJ, Liu H, Ridky TW, Cassarino D, Segal E, Chang HY, Cell Stem Cell. 2008;2:333-344.

[30] Monaghan JR, Walker JA, Page RB, Putta S, Beachy CK, Voss SR, J. Neurochem. 2007;101:27-40.

[31] Boyle KA, Traktman P, J. Virol. 2004;78:1992-2005.

[32] Stewart ZA, Pietenpol JA, Chem Res Toxicol. 2001;14:243-263.

[33] Taylor WR, Stark GR, Oncogene. 2001;20:1803-1815.

[34] Okorokov AL, Cell Cycle. 2003;2:233-235.

[35] Meek DW, Nat Rev Cancer. 2009;9:714-723.

[36] Saito S, Yamaguchi H, Higashimoto Y, Chao C, Xu Y, Fornace AJ, Jr., Appella E, Anderson CW, J. Biol. Chem. 2003;278:37536-37544.

[37] Toledo F, Wahl GM, Nat Rev Cancer. 2006;6:909-923.

[38] Kussie PH, Gorina S, Marechal V, Elenbaas B, Moreau J, Levine AJ, Pavletich NP, Science. 1996;274:948-953. 
[39] Schon O, Friedler A, Bycroft M, Freund S, Fersht A, J. Mol. Biol. 2002;323:491501.

[40] Teufel DP, Bycroft M, Fersht AR, Oncogene. 2009;28:2112-2118.

[41] Lee CW, Ferreon JC, Ferreon AC, Arai M, Wright PE, Proc. Natl. Acad. Sci. U S A. 2010.

[42] Riley T, Sontag E, Chen P, Levine A, Nat Rev Mol Cell Biol. 2008;9:402-412.

[43] Zhang XP, Liu F, Cheng Z, Wang W, Proc. Natl. Acad. Sci. U S A.

2009;106:12245-12250.

[44] Moll UM, Petrenko O, Mol Cancer Res. 2003;1:1001-1008.

[45] Lahav G, Adv. Exp. Med. Biol. 2008;641:28-38.

[46] Lahav G, Rosenfeld N, Sigal A, Geva-Zatorsky N, Levine AJ, Elowitz MB, Alon U, Nat. Genet. 2004;36:147-150.

[47] Li DW, Liu JP, Schmid PC, Schlosser R, Feng H, Liu WB, Yan Q, Gong L, Sun

SM, Deng M, Liu Y, Oncogene. 2006;25:3006-3022.

[48] Valbuena A, Vega FM, Blanco S, Lazo PA, Mol. Cell. Biol. 2006;26:4782-4793.

[49] Crighton D, Wilkinson S, O'Prey J, Syed N, Smith P, Harrison PR, Gasco M,

Garrone O, Crook T, Ryan KM, Cell. 2006;126:121-134.

[50] Valbuena A, Castro-Obregon S, Lazo PA, PLoS ONE. 2011;6:e17320.

[51] Waters K, Yang AZ, Reinke V, Dev. Biol. 2010;344:1011-1025.

[52] Segura-Totten M, Kowalski AK, Craigie R, Wilson KL, J. Cell. Biol.

2002;158:475-485.

[53] Bradley CM, Ronning DR, Ghirlando R, Craigie R, Dyda F, Nat. Struct. Mol. Biol. 2005;12:935-936.

[54] Margalit A, Brachner A, Gotzmann J, Foisner R, Gruenbaum Y, Trends Cell. Biol. 2007;17:202-208.

[55] Bengtsson L, Wilson KL, Mol. Biol. Cell. 2006;17:1154-1163.

[56] Lancaster OM, Cullen CF, Ohkura H, J. Cell. Biol. 2007;179:817-824.

[57] Goldfinger LE, Molecular bioSystems. 2008;4:293-299.

[58] Sanz-Garcia M, Lopez-Sanchez I, Lazo PA, Mol. Cell. Proteomics. 2008;7:21992214.

[59] Arnaoutov A, Dasso M, Dev. Cell. 2003;5:99-111.

[60] Gruss OJ, Carazo-Salas RE, Schatz CA, Guarguaglini G, Kast J, Wilm M, Le Bot N, Vernos I, Karsenti E, Mattaj IW, Cell. 2001;104:83-93.

[61] Kalab P, Pralle A, Isacoff EY, Heald R, Weis K, Nature. 2006;440:697-701.

[62] Carazo-Salas RE, Guarguaglini G, Gruss OJ, Segref A, Karsenti E, Mattaj IW,

Nature. 1999;400:178-181.

[63] Clarke PR, Zhang C, Nat Rev Mol Cell Biol. 2008;9:464-477.

[64] Johansen KM, Johansen J, Chromosome Res. 2006;14:393-404.

[65] Georgatos SD, Markaki Y, Christogianni A, Politou AS, Front. Biosci.

2009;14:2017-2027.

[66] Ivanovska I, Orr-Weaver TL, Cell cycle (Georgetown, Tex. 2006;5:2064-2071.

[67] Brittle AL, Nanba Y, Ito T, Ohkura H, Exp. Cell Res. 2007;313:2780-2785.

[68] Hauf S, Cole RW, LaTerra S, Zimmer C, Schnapp G, Walter R, Heckel A, van

Meel J, Rieder CL, Peters JM, J. Cell. Biol. 2003;161:281-294.

[69] Persico A, Cervigni RI, Barretta ML, Colanzi A, FEBS Lett. 2009;583:3857-3862.

[70] Acharya U, Mallabiabarrena A, Acharya JK, Malhotra V, Cell. 1998;92:183-192.

[71] Colanzi A, Sutterlin C, Malhotra V, J. Cell. Biol. 2003;161:27-32.

[72] Shaul YD, Seger R, J. Cell. Biol. 2006;172:885-897.

[73] Xie S, Wang Q, Ruan Q, Liu T, Jhanwar-Uniyal M, Guan K, Dai W, Oncogene.

2004;23:3822-3829. 
[74] Archambault V, Glover DM, Nat. Rev. Mol. Cell. Biol. 2009;10:265-275.

[75] Colanzi A, Corda D, Curr. Opin. Cell Biol. 2007;19:386-393.

[76] Feinstein TN, Linstedt AD, Mol. Biol. Cell. 2007;18:594-604.

[77] Ivanovska I, Khandan T, Ito T, Orr-Weaver TL, Genes Dev. 2005;19:2571-2582.

[78] Wormbase,

http://www.wormbase.org/db/misc/biblio?name=WBGene00017895\&category=\&class

=Gene\&abstract=WBPaper00027981\#WBPaper00027981. 2009.

[79] Rodenas E, Klerkx EP, Ayuso C, Audhya A, Askjaer P, Dev. Biol. 2009;327:399409.

[80] Klerkx EP, Alarcon P, Waters K, Reinke V, Sternberg PW, Askjaer P, Dev. Biol. 2009;335:12-21.

[81] Wiebe MS, Nichols RJ, Molitor TP, Lindgren JK, Traktman P, Biol. Reprod. 2010;82:182-193.

[82] Choi YH, Park CH, Kim W, Ling H, Kang A, Chang MW, Im SK, Jeong HW, Kong YY, Kim KT, PLoS ONE. 2010;5:e15254.

[83] Schober CS, Aydiner F, Booth CJ, Seli E, Reinke V, Mech. Dev. 2011.

[84] Renbaum P, Kellerman E, Jaron R, Geiger D, Segel R, Lee M, King MC, LevyLahad E, Am. J. Hum. Genet. 2009;85:281-289.

[85] Hanahan D, Weinberg RA, Cell. 2011;144:646-674.

[86] Karaman MW, Herrgard S, Treiber DK, Gallant P, Atteridge CE, Campbell BT, Chan KW, Ciceri P, Davis MI, Edeen PT, Faraoni R, Floyd M, Hunt JP, Lockhart DJ, Milanov ZV, Morrison MJ, Pallares G, Patel HK, Pritchard S, Wodicka LM, Zarrinkar PP, Nat. Biotechnol. 2008;26:127-132.

[87] Zhang J, Yang PL, Gray NS, Nat Rev Cancer. 2009;9:28-39. 


\section{FIGURE LEGENDS}

Figure 1. Potential cell division processes where VRK1 might play a regulatory role.

Figure 2. Localization of human VRK1 in different stages of cell cycle progression in HeLa cells. In mitosis, VRK1 is not associated with condensed chromosomes.

Figure 3. Double autoregulatory loop between VRK1, p53 and the p53-induced gene expression targets mdm2/hdm2 and DRAM. The activation of $\mathrm{p} 53$ by phosphorylation induces two genes, hdm2/mdm2 and DRAM, that contribute to downregulation of p53 in the proteasome and of VRK1 in lysosomes. Based on Valbuena et al. [50] 